\title{
INVASIVE ASPERGILLOSIS IN INTENSIVE CARE UNIT PATIENTS IN IRAN
}

\author{
Mohammad T. Hedayati ${ }^{1,2}$, Sadegh Khodavaisy ${ }^{3}$, Masoud Alialy, Saeed Mahdavi Omran ${ }^{5}$, Mohammad R. Habibi \\ Mazandaran University of Medical Sciences, Sari, Iran: Department of Medical Mycology and Parasitology' Invasive \\ Fungi Research Center ${ }^{2}$, Pulmonary and Critical Care Division ${ }^{4}$, Anesthesiologist and Critical Care Division ${ }^{6}$; Kurdestan \\ University of Medical Sciences, Sanandaj, Iran: Department of Medical Mycology and Parasitology ${ }^{3}$; Babol University \\ of Medical Sciences, Babol, Iran: Department of Medical Mycology and Parasitology 5
}

Summary: We assessed the intensive care unit (ICU) patients for Invasive aspergillosis (IA) with culture and non-culture based diagnostic methods from Iran. Thirty-six ICU patients with underlying predisposing conditions for IA were enrolled in the study. Sixty eight Bronchoalveolar lavage (BAL) samples were collected by bronchoscope twice a weekly. BAL samples were analyzed by microscopic examination, fungal culture and galactomannan (GM) detection. The Platelia Aspergillus GM EIA was used to quantify GM indices. Samples with a BAL GM index $\geq 1$ were considered as positive for GM. Patients were classified as having probable or possible IA. Out of 36 suspected patients to IA, $36.1 \%$ of cases showed IA which were categorized as: 4 cases of possible IA and 9 of probable IA. $76.2 \%$ of BAL samples were positive for GM. From 13 patients with IA, $11(84.6 \%)$ had at least one positive BAL GM index. Of these patients, $9(81.8 \%)$ showed probable IA. The main underlying predisposing conditions were neutropenia (53.8\%) and COPD (30.8\%). Our study has indicated that COPD must be considered as one of the main predisposing condition for occurrence of aspergillosis in ICU patients. Our data have also revealed that GM detection in BAL samples play a significant role to IA diagnosis.

Key word: Invasive aspergillosis; ICU; GM detection; $B A L$

\section{Introduction}

Aspergillus is one of the most prevalent airborne fungi both in indoor and outdoor environment. Our previous studies have also shown that the equipment and medical devices, potted plant and water can be a significant source for Aspergillus distribution in hospitals (17-19). Inhalation of Aspergillus conidia can give rise to various clinical conditions, depending essentially on the host's immunological status (22). Invasive aspergillosis (IA) is one of the most common life-threatening fungal infections among critically ill patients including intensive care unit (ICU) patients. In the last two decades the infections caused by Aspergillus species have increased and Aspergillus is now the most common cause of systemic fungal infections in patients undergoing allogeneic bone marrow transplantation and those with acute leukaemia undergoing intensive chemotherapy $(5,26,31)$.

Recent investigations have shown that IA must be considered as an emerging and mortal infectious disease in ICU patients even in the absence of an apparent predisposing immunodeficiency $(24,25)$. In addition, several new publications have also indicated that frequency of IA in the ICU has increased $(13,27,30)$. On the other hand, IA in ICU patients is associated with prolonged hospital stay, and increased cost (30). The diagnosis of IA is still a great challenge in the ICU, and it is often made late in the course of the infection because of clinical manifestations are usually non-specific, mycological cultures are difficult to interpret or fungal growth is often not present even from patients diagnosed with fungal diseases, and invasive procedures require to obtain histological specimens $(8,27,35,38)$.

Galactomannan (GM) is a polysaccharide fungal cell wall component that is released during tissue invasion by Aspergillus hyphae and that can be detected in body fluids including BAL. GM detection in serum and BAL fluids as a means of establishing early diagnosis of IA in patients at risk is very promising. GM test shows a sensitivity that may range from $60 \%$ to $100 \%$ and specificity from $81 \%$ to $99 \%$ in neutropenic patients, depending of the tested sample (serum or BAL) and cut-off values $(14,36)$.

In Iran, there is no report on invasive fungal infections in ICU patients; however, some publications have focused on the Aspergillus as the causative agent of invasive infections in immunocompromised patients $(2,3)$. According to these facts, we assessed the ICU patients for IA with culture and non-culture based diagnostic methods from Iran.

\section{Material and Methods}

\section{Patients}

During one year, 818 admitted patients to our medical ICU were reviewed for inclusion in this prospective 
study. The study was approved by the ethics committee of Mazandaran University of Medical Sciences and written and informed consent was obtained from the patient or next of kin.

Patients who were older than 16 years and displayed at least one of the following host factors were enrolled in the study:

1. A hematologic malignancy, unless they were already treated with antifungals

2. Cancer and receiving chemotherapy within the last 3 months before admission

3. Neutropenia

4. Chronic obstructive pulmonary disease (COPD)

5. Solid organ transplant recipient

6. Steroid use: at least $4 \mathrm{mg}$ methylprednisolone (or equivalent) a day for at least 7 days in the past 3 weeks before admission or during the course of the ICU stay for at least 5 days or a cumulative dose of at least $250 \mathrm{mg}$ of methylprednisolone (or equivalent) in the past 3 months before enrollment

7. Recipient of any other immunosuppressive treatment (tacrolimus, cyclosporine, methotrexate, cyclophosphamide, sirolimus)

8. ICU stay more than 21 days

\section{Laboratory work}

Fiberoptic bronchoscopy (Olympus BF20D) with BAL (25-30 ml) was performed twice weekly thereafter if feasible. The sampling area was selected based on the infiltrate location on the chest radiograph. The presence of any tracheal or bronchial lesions was recorded by the endoscopist. A total of sixty eight BAL fluid samples were collected. BAL samples were not obtained from 5 patients due to their critically ill conditions or death, in second sampling time. BAL samples were analyzed by direct microscopic examination using CalcoFlour White staining, fungal culture and GM detection. Cultures for fungi were performed by inoculating clinical specimens onto Sabouraud glucose agar at $30{ }^{\circ} \mathrm{C}$ for 10 days. The fungi were identified by standard mycological techniques based upon gross cultural and microscopic morphology.

The PLATELIATM ASPERGILLUS EIA (Bio-Rad, France) was used to quantify GM indices as per the manufacturer's instructions. BAL fluid samples were processed under a high-efficiency particulate air-filtered hood. Briefly, samples were mixed well and $300 \mu$ of each was added to $100 \mu \mathrm{l}$ of $4 \%$ EDTA treatment solution, boiled for $3 \mathrm{~min}$, and centrifuged at $10,000 \mathrm{rpm}$ for $10 \mathrm{~min}$. Supernatant $(50 \mu \mathrm{l})$ was added to $50 \mu \mathrm{l}$ of a reaction mixture containing conjugated anti-GM EB-A2 antibody, and the mixture was incubated in microtiter plates precoated with the same antibody (EB-A2) for $90 \mathrm{~min}$ at $37^{\circ} \mathrm{C}$. Wells were washed with an automated washer (SCO Diagnostics washer MPW1, Germany) and incubated with $200 \mu$ l of tetramethylbenzidine solution for an additional $30 \mathrm{~min}$ in the dark. Reactions were stopped with $100 \mu \mathrm{l}$ of $1.5 \mathrm{M}$ sulfuric acid, and optical densities (ODs) at 450 and $620 \mathrm{~nm}$ were read. Positive and negative controls (provided in the kit) were included in each assay. Results were recorded as an index relative to the OD of the threshold control (GM index $=\mathrm{OD}$ sample/OD threshold control). Samples with a BAL GM index $\geq 1$ were considered as positive for GM.

\section{Criteria for probable or possible IA}

Patients were classified as having probable or possible IA, based on the standardized Invasive Fungal Infections Group of the European Organization for the Research and Treatment of Cancer/ Mycoses Study Group (EORTC/ MSG) case definitions (9), with the modification that COPD, Stay in ICU more than 21 days and steroids were added to the host factor section. Detection of GM in two successive collected BAL samples was included as a microbiological criterion. In this present study we were not be able to define a proven IA case because there was an explicit refusal of the family to doing biopsy or autopsy. Probable IA referred to the presence of a host factor, a clinical criterion, and a mycological criterion. Possible IA was defined by the presence of a host factor and either a mycological criterion or one major (or two minor) clinical criteria.

\section{Results}

Out of 818 admitted patients to our ICU, 36 patients (4.4\%) fulfilling the inclusion criteria were enrolled. Out of all patients enrolled, $53.7 \%$ were men. The age range of the patients was $18-88$ years. The mean length of stay in ICU for suspected IA patients was 19.6 days.

Out of 36 suspected patients to IA, 13 cases (36.1\%) showed IA. According to criteria presented by EORTC/ MSG, they were categorized as: 4 cases $(30.8 \%)$ of possible IA and $9(69.2 \%)$ of probable IA. Table 1 shows the direct microscopic examination, culture and GM detection findings in BAL samples as well as underlying conditions, type of IA and outcome of disease in ICU patients with IA. A total of 21 BAL samples from patients with IA were analyzed by microscopic and culture methods; 18 (85.7\%) and $11(52.4 \%)$ of these samples were positive for septate hyphae and Aspergillus growth, respectively. Among Aspergillus species, A. flavus and A. fumigatus were equally isolated from positive samples for Aspergillus growth. Of 21 BAL samples, 16 (76.2\%) were positive for GM. Other collected BAL samples had GM index $\geq 0.5$ and $<1.0$. Of 21 BAL samples, $6(28.6 \%)$ samples that were negative for Aspergillus growth on culture had GM index $<1.0$. From 13 patients with IA, 11(84.6\%) had at least one positive BAL GM index. Of these patients, $9(81.8 \%)$ showed probable IA. Five patients $(38.5 \%)$ were positive for GM in both sampling times. Three patients who were positive for GM in first BAL sample died before second sampling time. The main underlying predisposing conditions were neutrope- 
Tab. 1: Data for ICU patients with probable or possible aspergillosis included in this study $(\mathrm{n}=13)$

\begin{tabular}{|c|c|c|c|c|c|c|c|c|c|}
\hline \multirow[t]{2}{*}{ Patient } & \multicolumn{2}{|c|}{$\begin{array}{l}\text { Microscopic } \\
\text { results }\end{array}$} & \multicolumn{2}{|c|}{ Culture results } & \multicolumn{2}{|c|}{$\begin{array}{l}\text { BAL GM } \\
\text { results }\end{array}$} & \multirow[t]{2}{*}{$\begin{array}{l}\text { Underlying } \\
\text { condition }\end{array}$} & \multirow[t]{2}{*}{ IA } & \multirow[t]{2}{*}{ Outcome } \\
\hline & $\begin{array}{l}\text { First } \\
\text { sample }\end{array}$ & $\begin{array}{l}\text { Second } \\
\text { sample }\end{array}$ & $\begin{array}{l}\text { First } \\
\text { sample }\end{array}$ & $\begin{array}{l}\text { Second } \\
\text { sample }\end{array}$ & \begin{tabular}{|l} 
First \\
sample
\end{tabular} & $\begin{array}{l}\text { Second } \\
\text { sample }\end{array}$ & & & \\
\hline 1 & $\mathrm{H}$ & - & $\begin{array}{l}\text { A. } \\
\text { fumigatus }\end{array}$ & - & 3.3 & - & Neutropenia & probable & Death \\
\hline 2 & $\mathrm{H}$ & $\mathrm{H}$ & $\mathrm{Neg}$ & $\mathrm{Neg}$ & 0.6 & 0.8 & Neutropenia & possible & Survived \\
\hline 3 & $\mathrm{H}$ & $\mathrm{H}$ & $\begin{array}{l}\text { A. } \\
\text { fumigatus }\end{array}$ & $\begin{array}{l}\text { A. } \\
\text { fumigatus }\end{array}$ & 3.2 & 3.7 & COPD & possible & Death \\
\hline 4 & $\mathrm{H}$ & $\mathrm{H}$ & $\mathrm{Neg}$ & $\mathrm{Neg}$ & 0.6 & 1.1 & Neutropenia & probable & Death \\
\hline 5 & $\mathrm{H}$ & $\mathrm{H}$ & $\begin{array}{l}\text { A. } \\
\text { fumigatus }\end{array}$ & $\begin{array}{l}\text { A. } \\
\text { fumigatus }\end{array}$ & 2.6 & 1.2 & COPD & probable & Death \\
\hline 6 & Neg & $\mathrm{Neg}$ & $\mathrm{Neg}$ & $\mathrm{Neg}$ & 0.7 & 0.5 & COPD, Diabetes & possible & Survived \\
\hline 7 & $\mathrm{H}$ & - & A. flavus & - & 2.3 & - & $\begin{array}{l}\text { Hematologic } \\
\text { malignancy }\end{array}$ & probable & Death \\
\hline 8 & $\mathrm{H}$ & $\mathrm{H}$ & $\begin{array}{l}\text { A. } \\
\text { fumigatus }\end{array}$ & $\begin{array}{l}\text { A. } \\
\text { fumigatus }\end{array}$ & 2.8 & 3.6 & Neutropenia & probable & Survived \\
\hline 9 & $\mathrm{H}$ & $\mathrm{H}$ & Neg & Neg & 2.8 & 2.7 & $\begin{array}{l}\text { COPD, ICU stay } \\
\text { longer than } 21 \text { days }\end{array}$ & possible & Death \\
\hline 10 & $\mathrm{H}$ & - & A. flavus & - & 3.2 & - & $\begin{array}{l}\text { Hematologic } \\
\text { malignancy }\end{array}$ & probable & Survived \\
\hline 11 & - & $\mathrm{H}$ & A. flavus & A. flavus & 3.3 & 2.6 & Neutropenia & probable & Death \\
\hline 12 & $\mathrm{H}$ & - & $\mathrm{Neg}$ & - & 2.9 & - & Neutropenia & probable & Death \\
\hline 13 & $\mathrm{H}$ & - & $\mathrm{Neg}$ & - & 3.2 & - & Neutropenia & probable & Survived \\
\hline
\end{tabular}

nia (53.8\%), COPD (30.8\%) and hematologic malignancy $(15.4 \%)$. Of 13 patients with IA, Eight $(61.5 \%)$ died. The mortality percentage among patients with probable and possible IA was $66.7 \%$ and $50 \%$, respectively.

\section{Discussion}

In this present study, we presented a population of 818 ICU patients of which $4.4 \%$ had host factor, clinical or mycological criteria for infection with Aspergillus. This rate is in line with data of previous studies (24). Out of 36 cases of our ICU patients who met criteria for Aspergillus infection, $36.1 \%$ showed IA. The mortality percentage among IA patients was $61.5 \%$. The previous studies have shown a diverse incidence of IA in ICU patients $(10,24$, 37). Meersseman et al. (24) in a review paper presented an incidence of IA from $0.33 \%$ to $19 \%$ among ICU patients. Recently, Tortorano et al. (37) in a prospective survey in 38 ICUs of 27 Italian hospitals reported the median rate of 6.31 per 1000 admissions (range 1.26-28.64) for Aspergillus infection. This diversity may be due to difference in design of study, duration of study, type of underlying or immunosuppressive conditions and diagnosis methods. In addition, some authors suggested that invasive fungal in- fections are among the most commonly missed diagnoses in ICU patients $(7,28,34)$.

In the present study we applied GM detection in BAL as one of the mycological criteria. However the Platelia Aspergillus GM EIA was designed to detect the presence of GM in serum samples but some authors suggested that it can be beneficial to detect GM in BAL fluid for IA diagnosis with high sensitivities and specificities $(6,20,32)$. The previous studies $(4,19,20)$ have shown that a GM index of $\geq 1.0$ in comparison with 0.5 in BAL fluid enhances the diagnosis of IA in patients who are at risk; so in the present study, we used this GM index as a cutoff value for positive BAL GM.

The mortality percentage in our study was $66.7 \%$ and $50 \%$ among patients with probable and possible IA, respectively. These data are concordant with other previous studies $(10-12,24)$ however Meersseman et al. (25) reported a mortality rate for IA in ICU patients up to $97 \%$. It should also be indicated that aspergillosis is more severe than candidiasis as the most prevalent fungal infections among ICU patients (37).

Eleven Of 21 BAL samples (52.4\%) from IA patients were positive both for GM and for Aspergillus growth on culture. On the other hand, six samples $(28.6 \%)$ that were 
negative for Aspergillus growth on culture had GM index $<1.0$. This results show that the detection of GM in BAL fluid enhances the identification of Aspergillus species as a cause of pulmonary disease in ICU patients. The result of several studies have also shown that the GM analysis of BAL samples should consider for IA diagnosis among high-risk patients, especially for the ease of processing and the more rapid result than traditional methods $(1,23,29)$. Moreover, all patients with probable IA had at least one positive BAL GM index, in the present study. This result can account for the importance of GM detection in the process of IA.

In the present study $A$. fumigatus and A. flavus were equally isolated from the BAL samples of patients with IA. In most previous studies, $A$. fumigatus was the predominant species of Aspergillus from patients with IA (10, 21, 33, 37). In our previous environmental studies $A$. flavus also was the most distributed species among genus Aspergillus in indoor and outdoor environment $(16,17)$. More distribution of $A$. flavus in environment can facilitate exposure and increase the risk of the colonization with this species.

In this present study the main underlying predisposing factors was neutropenia followed by COPD and hematologic malignancy. However neutropenia was reported as the main predisposing factor for IA but patients with COPD have emerged to be at particular risk for IA (10, $15,25,30)$. Because of long-term corticosteroid use by COPD patients, which has significant impact on the distribution and function of neutrophils, macrophages, and lymphocytes, COPD can be considered as one of the main predisposing factors for IA.

\section{Conclusion}

The results of our study showed that IA is a life threatening infectious disease in ICU. Our study has also indicated that non-classical risk factors especially COPD must be considered as a main predisposing condition for occurrence of aspergillosis in ICU patients. Our data have also revealed that GM detection in BAL samples play a significant role to IA diagnosis.

\section{Acknowledgements}

This work was supported by grants from the Mazandaran University of Medical Sciences.

\section{References}

1. Acosta J, Catalan M, del Palacio-Peréz-Medel A, et al. A prospective comparison of galactomannan in bronchoalveolar lavage fluid for the diagnosis of pulmonary invasive aspergillosis in medical patients under intensive care: comparison with the diagnostic performance of galactomannan and of $(1 \rightarrow 3)$ - $\beta$-d-glucan chromogenic assay in serum samples. Clin Microbiol Infect 2011; 17(7): 1053-60.

2. Badiee $P$, Alborzi A. Invasive fungal infections in renal transplant recipients. Exp Clin Transplant 2011; 9(6): 355-62

3. Badiee P, Kordbacheh P, Alborzi A, Zeini F, Mirhendi H, Mahmoody M. Fungal infections in solid organ recipients. Exp Clin Transplant 2005; 3(2): 385-9.
4. Becker MJ, Lugtenburg EJ, Cornelissen JJ, van der Schee C. Galactomannan detection in computerized tomography-based bronchoalveolar lavage fluid and serum in haematological patients at risk for invasive pulmonary aspergillosis. Brit J Haematol 2003; 121: 448-457.

5. Chamilos G, Luna M, Lewis RE, et al. Invasive fungal infections in patients with hematologic malignancies in a tertiary care cancer center: an autopsy study over a 15-year period (1989-2003). Haematologica 2006; 91(7): 986-9.

6. Clancy CJ, Jaber RA, Leather LH, et al. Bronchoalveolar lavage galactomannan in diagnosis of invasive pulmonary aspergillosis among solid-organ transplant recipients. J Clin Microbiol 2007; 45: 1759-65.

7. Combes A, Mokhtari M, Couvelard A, et al. Clinical and autopsy diagnoses in the intensive care unit: a prospective study. Arch Intern Med 2004; 164: 389-92.

8. Denning DW, Park S, Lass-Florl C, Fraczek MG, et al. High-frequency triazole resistance found In nonculturable Aspergillus fumigatus from lungs of patients with chronic fungal disease. Clin Infect Dis 2011; 52(9): 1123-9.

9. De Pauw B, Walsh TJ, Donnelly JP, et al. Revised definitions of invasive fungal disease from the European Organization for Research and Treatment of Cancer/ Invasive Fungal Infections Cooperative Group and the National Institute of Allergy and Infectious Diseases Mycoses Study Group (EORTC/MSG) Consensus Group. Clin Infect Dis. 2008 Jun 15; 46(12): 1813-21.

10. Dutkiewicz R, Hage CA. Aspergillus infections in the critically ill. Proc Am Thorac Soc 2010; 7(3): 204-9.

11. Garbino J, Fluckiger U, Elzi L, Imhof A, Bille J, Zimmerli S. Survey of aspergillosis in non-neutropenic patients in Swiss teaching hospitals. Clin Microbiol Infect 2011; 17(9): 1366-71.

12. Garnacho-Montero J, Amaya-Villar R, Ortiz-Leyba C, et al. Isolation of Aspergillus spp. from the respiratory tract in critically ill patients: risk factors, clinical presentation and outcome. Crit Care 2005; 9(3): R191-9.

13. Glöckner A, Karthaus M. Current aspects of invasive candidiasis and aspergillosis in adult intensive care patients. Mycoses 2011; 54(5): 420-33.

14. Guinea J, Jensen J, Peláez T, et al. Value of a single galactomannan determination (Platelia) for the diagnosis of invasive aspergillosis in nonhematological patients with clinical isolation of Aspergillus spp. Med Mycol 2008; 46: 575-579.

15. Guinea J, Torres-Narbona M, Gijón P, et al. Pulmonary aspergillosis in patients with chronic obstructive pulmonary disease: incidence, risk factors, and outcome. Clin Microbiol Infect 2010; 16(7): 870-7.

16. Hedayati MT, Mayahi S, Denning DW. A study on Aspergillus species in houses of asthmatic patients from Sari city, Iran and a brief review of the health effects of exposure to indoor Aspergillus. Environ Monit Assess 2010; 168: 481-487.

17. Hedayati MT, Mayahi S, Movahedi M, Shokohi T. A Study on Fungal Flora of Tap Water as a Potential Reservoir of Fungi in Hospitals from Sari city, Iran. J Mycol Méd 2011; 21: 10-14.

18. Hedayati MT, Mohammadpour RA. A survey on the mycological contamination of the air and the equipment of operating rooms of 17 hospitals. J Med faculty Gilan Univ Med Sci 1999; 8(19): 56-61.

19. Hedayati MT, Mohseni-Bandpi A, Moradi S. A survey on the pathogenic fungi in soil samples of potted plants from Sari hospitals, Iran. J Hosp Infect 2004; 58: $59-62$.

20. Husain S, Clancy CJ, Nguyen MH, et al. Performance characteristics of the Platelia Aspergillus enzyme immunoassay for detection of Aspergillus galactomannan antigen in bronchoalveolar lavage fluid. Clin Vaccine Immunol 2008; 15: 1760-3.

21. Khasawneh F, Mohamad T, Moughrabieh MK, Lai Z, Ager J, Soubani AO. Isolation of Aspergillus in critically ill patients: a potential marker of poor outcome. J Crit Care 2006 Dec; 21(4): 322-7.

22. Kousha M, Tadi R, Soubani AO. Pulmonary aspergillosis: a clinical review. Eur Respir Rev 2011; 20(121): 156-74.

23. Maertens J, MaertensV, Theunissen K, et al. Bronchoalveolar Lavage Fluid Galactomannan for the Diagnosis of Invasive Pulmonary Aspergillosis in Patients with Hematologic Diseases. Clin Infect Dis 2009; 49: 1688-93.

24. Meersseman W, Lagrou K, Maertens J, Van Wijngaerden E. Invasive aspergillosis in the intensive care unit. Clin Infect Dis 2007; 45(2): 205-16.

25. Meersseman W, Vandecasteele SJ, Wilmer A, Verbeken E, Peetermans WE, Van Wijngaerden E. Invasive aspergillosis in critically ill patients without malignancy. Am J Respir Crit Care Med 2004; 170: 621-625.

26. Mikulska M, Raiola AM, Bruno B, et al. Risk factors for invasive aspergillosis and related mortality in recipients of allogeneic SCT from alternative donors: an analysis of 306 patients. Bone Marrow Transplant 2009; 44(6): 361-70.

27. Morace G, Borghi E. Fungal infections in ICU patients: epidemiology and the role of diagnostics. Minerva Anestesiol 2010; 76(11): 950-6.

28. Mort TC, Yeston NS. The relationship of pre mortem diagnoses and post mortem findings in a surgical intensive care unit. Crit Care Med 1999; 27: 299-303.

29. Pasqualotto AC, Xavier MO, Sánchez LB, et al. Diagnosis of invasive aspergillosis in lung transplant recipients by detection of galactomannan in the bronchoalveolar lavage fluid. Transplantation. 2010; 90(3): 306-11.

30. Patel DA, Gao X, Stephens JM, Forshag MS, Tarallo M. US hospital database analysis of invasive aspergillosis in the chronic obstructive pulmonary disease non-traditional host. J Med Econ 2011; 14(2): 227-37.

31. Richardson MD. Changing patterns and trends in systemic fungal infections. J Antimicrob Chemother 2005; 56 Suppl 1: i5-i11. 
32. Sanguinetti M, Posteraro B, Pagano L, et al. Comparison of real-time PCR, conventional PCR, and galactomannan antigen detection by enzyme- linked immunosorbent assay using bronchoalveolar lavage fluid samples from hematology patients for diagnosis of invasive pulmonary aspergillosis. J Clin Microbiol 2003 41: 3922-5.

33. Shoham S, Marwaha S. Invasive fungal infections in the ICU. J Intensive Care Med 2010; 25(2): 78-92.

34. Silfvast T, Takkunen O, Kolho E, Andersson L, Rosenberg P. Characteristics of discrepancies between clinical and autopsy diagnoses in the intensive care unit: a 5-year review. Intensive Care Med 2003; 29: 321-4.
35. Smith JA, Kauffman CA. Recognition and prevention of nosocomial invasive fungal infections in the intensive care unit. Crit Care Med 2010; 38(8 Suppl): S380-7.

36. Thornton CR. Detection of invasive aspergillosis. Adv Appl Microbiol 2010; 70: $187-216$.

37. Tortorano AM, Dho G, Prigitano A, et al. Invasive fungal infections in the intensive care unit: a multicentre, prospective, observational study in Italy (20062008). Mycoses 2012; 55(1): 73-79.

38. Zilberberg MD, Shorr AF. Fungal infections in the ICU. Infect Dis Clin North Am 2009; 23(3): 625-42.

Received: 18/01/2013

Accepted in revised form: 07/06/2013

\section{Correspondence author:}

Dr Mohammad T. Hedayati, Department of Medical Mycology and Parasitology, School of Medicine, Mazandaran University of Medical Sciences, Kilometer 18 Khazarabad Road, P.O. Box 48175-1665, Sari, Iran; e-mail: hedayaty2001 @yahoo.co.uk 